\title{
DNA electrodes for detection of sequence specific nucleic acid-ligand interaction
}

\author{
C. Tersch ${ }^{1}$, C. Witte ${ }^{1}$, J. Glöckler ${ }^{2}$, F. Lisdat ${ }^{1}$ \\ ${ }^{1}$ Biosystems Technology, Wildau Technical University of Applied Sciences \\ Bahnhofstr. 1, 15745 Wildau, Germany, \\ ${ }^{2}$ Alacris Theranostics GmbH/ MPI for Molecular Genetics, Ihnestr. 63, 14195 Berlin, Germany \\ e-mail flisdat@th-wildau.de
}

\begin{abstract}
In this study an impedimetric DNA sensor has been used for the detection of protein-DNA interactions. The sensor is based on single-stranded DNA bound to gold chip electrodes. In the presence of ferri/ferrocyanide impedance measurements show an increase in charge transfer resistance Rct after immobilization and hybridization of ssDNA to the sensor surface. This allows hybridization detection in the low nanomolar concentration range. This electrode system is used to follow the sequence specific reaction of the restriction endonuclease $\mathrm{BamHI}$ on the surface electrochemically. Furthermore, the sequence specific DNA-binding of the transcription factor NF-KB p50 is found to cause a decrease in Rct resistance in a concentration dependent way.
\end{abstract}

Key words: DNA electrode, impedance, voltammetry, SPR, DNA-protein binding

\section{Motivation}

The detection of proteins and low molecular weight compounds interacting specifically with nucleic acids has become an interesting research target. These reactions are important for many biochemical processes inside cells but also in drug development. Methodically labelfree techniques have become increasingly popular since they allow a direct detection with non-modified biomolecules. Impedimetric analysis of DNA hybridization has been successfully studied $[1,2]$ and thus we want to investigate the potential use of this method for analytical detection of biomolecular interactions.

\section{Results}

The sensor consists of short thiol-modified single-stranded DNA, which is chemisorbed to gold chips and a short mercaptobutanol layer preventing non-specific interactions. Impedimetric analysis in the presence of a redox couple show that the charge transfer resistance Rct is a very sensitive parameter to follow the DNA immobilization, hybridization and binding of molecules to DNA. Capacitive changes are rather small [3]. The surface concentration can be adjusted during the immobilization and controlled with a complementary strand with a covalently fixed redox label (methylene blue) and voltammetric analysis. For DNA detection by hybridization the surface concentration turned out be an important factor governing the sensitivity of the
Impedimetric detection. Response of nanomolar nucleic acid concentrations can be achieved.

DNA of different length is used in order to provide better accessibility for protein interaction.

The charge situation on the surface turns out to be very important for the resistive properties. This can be verified e.g. by the positively charged groove binder spermine. Protein binding to SSDNA or dsDNA can be detected with single strand binding protein (SSBP) and a DNA antibody.

The electrode system is used to follow an enzyme reaction on the surface electrochemically. The enzymatic cleavage of a double-stranded DNA by BamHI endonuclease could be followed by impedance spectroscopy (Fig.1). For verification it is also analyzed by cyclic voltammetry with labeled DNA. Specificity can be demonstrated using non-specific sequences (Fig.2).

Furthermore, the sequence specific binding of the transcription factor NF-KB p50-homodimer on dsDNA with the recognition sequence is found to cause a decrease in charge transfer resistance. This signal change occurs due to a neutralization effect of the negatively charged DNA backbone (Fig.3). Specific protein binding can be detected in the concentration range from $4-23 \mu \mathrm{g} / \mathrm{ml}$ [4]. This work represents some basic experiments for the label free impedimetric detection of protein-DNA interactions. The monitoring of changes in the electrical properties of the sensing interface by impedance spectroscopy has potential for a 
simple, rapid and low-cost analysis. When DNA is immobilized on thin gold films this can be also used for a label free detection by SPR. However the binding of low molecular weigth molecules is difficult to detect. The

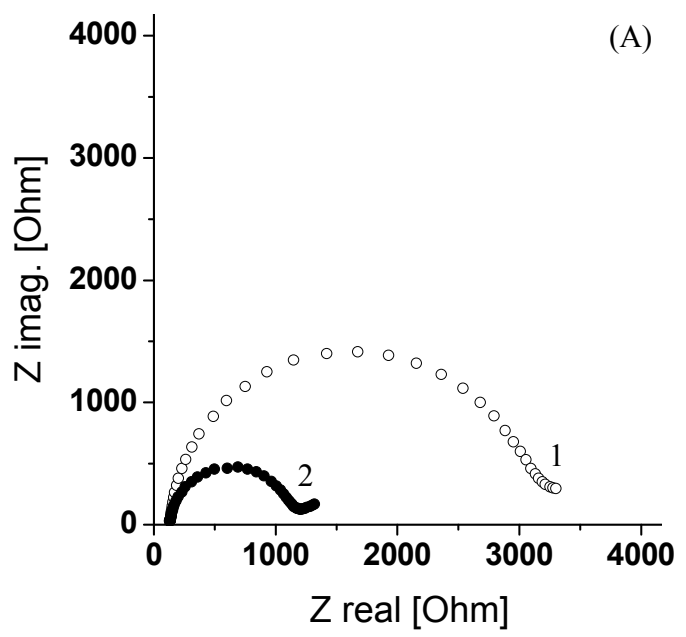

Fig. 1: Impedance spectra of an oligonucleotide electrode (26mer) with specific dsDNA before (1) and after (2) incubation in $1 \mathrm{U} / \mu \mathrm{l} \mathrm{BamHI}$ endonuclease.

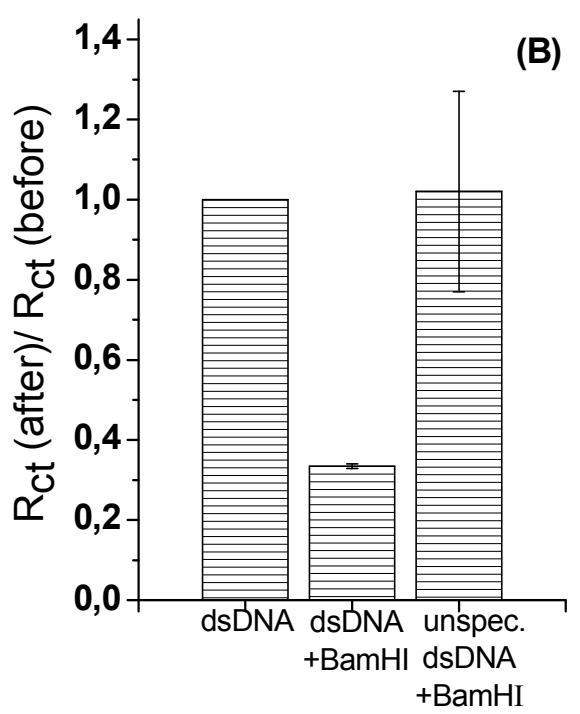

Fig.2 Ratio of the charge transfer resistance $R_{c t}$ (after) / $R_{c t}$ (before) determined after and before incubation with BamHI cleavage mixture on electrodes with dsDNA with the specific recognition sequence for BamHI or on dsDNA without the specific recognition sequence. (0.1 M sodium phosphate buffer $\mathrm{pH}$ 7, $2 \mathrm{mM}$ ferri-/ferrocyanide, error bars are results of three sensor preparations)

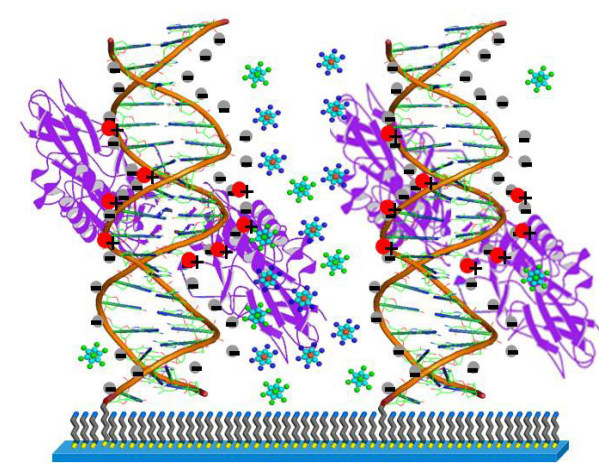

Fig. 3: Schematic illustration of NF-kB p50 interaction with the electrode fixed DNA and the change in charge situation on the surface.

presentation will also show the possibilities of using very long, repetitive DNA sequences as recognition layer which can cause strong conformational changes upon analyte interaction [5]. This results in larger changes in surface properties (e.g. optical density) and thus can be easily detected in a label free format. Consequently small molecules such as ions can be analysed without any amplification step.

\section{Acknowledgements}

This work was partially supported by the BMBF Germany (project 13N9593).

\section{References}

[1] R S. Pan, L. Rothberg, Langmuir 21(3) 1022 (2005), doi: 10.1021/la048083a

[2] J. Kafka, O. Pänke, B. Abendroth, F. Lisdat, Electrochim. Acta 53(25) (2008) 7467,

[3] doi: 10.1016/j.electacta.2008.01.031

[4] C. Witte, F. Lisdat, Electroanal. 23(2) 339 (2011), doi: 10.1002/elan.201000410

[5] C. Tersch, F. Lisdat, Electrochim. Acta 56(22) 7673 (2011) doi 10.1016/j.electacta.2011.06.063

[6] C. Schlachter, F. Lisdat, M. Frohme, V. A. Erdmann, Z. Konthur, H. Lehrach, J. Glökler, Biosensors Bioelectronics 31571 2012, doi: 10.1016/j.bios.2011.11.003 